\title{
Response to Hajnal et al.
}

\author{
Jon F. Davis • Stephen C. Benoit \\ Published online: 7 May 2013 \\ (C) Springer Science+Business Media New York 2013
}

This letter is in direct response to the editorial comment received from Hajnal et al., regarding our manuscript published in Obesity Surgery entitled "Roux en Y Surgery Increases Ethanol Consumption in the Rat" authored by myself, Andrea Tracy, Jennifer Schurdak, Irwin Magrisso, Bernadette Grayson, Randy Seeley, and Stephen Benoit. Before addressing Hajnal's specific concerns, I will first mention that we appreciate the interest our manuscript has received from Hajnal et al. The intention of this letter is to address thoroughly and completely the issues brought forth by Hajnal and colleagues.

Overall, the issue raised by Hajnal et al. is derived from editorial concerns rather than discrepancy in the reported findings. Dr. Andras Hajnal and colleagues are concerned with the inclusion of particular citations which support the overall conclusion of our manuscript, that RYGB surgery increases ethanol intake in rodents. Moreover, Hajnal and colleagues express additional comments regarding the failure to cite our own work which indicated that RYGB surgery leads to decreases in ethanol consumption in individuals who consume high levels of ethanol prior to surgery [2]. In our view, these are separate phenomena dictated by individual predisposition to consume ethanol prior to surgery and are in fact the subject of ongoing work in our laboratory. Therefore, I will not use this as a forum to discuss our ongoing studies.

In general, we certainly agree that including citations germane to this work are critical for the broader scientific community as well as those who closely study this particular area. In fact, we made every effort to carefully review the literature on this topic, which was scarce, before submitting either one of our manuscripts describing surgical effects on ethanol intake. However, as a point of clarification, the citation of the work of Ertelt mentioned by Hajnal et al. [1] was in fact referenced in

\section{J. F. Davis $(\square)$}

Department of Psychiatry, Metabolic Diseases Institute,

The University of Cincinnati, 2170 East Galbraith Road,

Cincinnati, OH 45237, USA

e-mail: jon.davis@uc.edu

S. C. Benoit

Department of Psychiatry and Behavioral Neuroscience, University of Cincinnati College of Medicine, 2170 Galbraith Road, Cincinnati, OH 45237, USA our manuscript. It is also our obligation to note plainly here that there were circumstances outside our control which ultimately prohibited us from providing a more current background of this work, which subsequently became the subject of the inquiry by Hajnal et al. Importantly, our manuscript was composed in fall of 2011, before many of the citations presented by Hajnal et al. were published. Moreover, the review process for our manuscript in question extended beyond 1 year, thus adding further delay before it was published. The latter seems particularly relevant to this discussion as many of the citations presented by Hajnal et al. were published in 2012 including our own. As previously stated, our manuscript was written in fall 2011 and submitted in January 2012. Following initial review, our manuscript was accepted in April 2012. The production of this accepted manuscript was voluntarily delayed by our group to validate accuracy of reported methods which resulted in our manuscript being resubmitted with minimal methodological changes in October 2012. Unfortunately due to confusion between the journal and the production team, we did not learn that our manuscript was accepted until February 2013 after a personal email to the editor. Therefore due to the aforementioned circumstance, the citations listed by Hajnal and colleagues were not included in our Obesity Surgery manuscript. Thank you for giving us the opportunity to respond to this inquiry and we appreciate the interest our manuscript has already generated.

Sincerely,

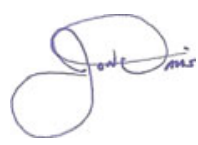

Jon F. Davis, PhD, Research Scientist

\section{References}

1. Ertelt TW et al. Alcohol abuse and dependence before and after bariatric surgery: a review of the literature and report of a new data set. Surg Obes Relat Dis. 2008;4(5):647-50.

2. Davis JF et al. Gastric bypass surgery attenuates ethanol consumption in ethanol-preferring rats. Biol Psychiatry. 2012;72(5):354-60. 